\title{
Antimicrobial Activity of Polyalthia longifolia (Sonn.) Thw. var. Pendula Leaf Extracts Against 91 Clinically Important Pathogenic Microbial Strains
}

\author{
Sumitra Chanda*, Rathish Nair \\ Phytochemical, Pharmacological and Microbiological Laboratory \\ Department of Biosciences, Saurashtra University, Rajkot, Gujarat, India \\ E-mail: svchanda@gmail.com \\ Received May 21, 2010; revised July 21, 2010; accepted July 26, 2010
}

\begin{abstract}
The methanol, acetone and 1,4-dioxan fractions of leaves of Polyalthia longifolia (Sonn.) Thw. were evaluated for antibacterial and antifungal activity. 91 clinically important strains were used for the study which were both clinical isolates as well as identified strains. Piperacillin and gentamicin were used as standards for antibacterial assay, while nystatin and flucanazole were used as standards for antifungal assay. The antibacterial activity was more pronounced against gram positive bacterial and fungal strains. Poor activity was shown against gram negative bacterial strains studied.
\end{abstract}

Keywords: Antibacterial, Antifungal, Polyalthia longifolia, Clinical Isolates, Organic Solvent Extracts

\section{Introduction}

Due to the increasing development of drug resistance in human pathogens as well as the appearance of undesirable effect on certain antimicrobial agents, there is a need to search for new agents. The world health organization in 1997 suggested that effective locally available plants be used as substitutes for drugs. Research work on medicinal plants be intensified and information on these plants be exchanged. This thought will go a long way in the scientific exploration of medicinal plants for the benefit of man and is likely to decrease the dependence on importance of drugs [1]. Polyalthia longifolia (Annonaceae) is a tree, which is widely distributed in Bangladesh, Srilanka and throughout the hotter parts of India [2]. In India, the seeds of this plant were used as febrifuge [3]. Literature survey revealed that most of the plants of annonaceae family contain antitumor and anticancer principles $[4,5]$. The bark is also used as a febrifuge in the Balasore district of Orissa [6]. The extract of stem bark and the alkaloids isolated from this were found to demonstrate a good antibacterial and antifungal activities [7]. In the present study, antimicrobial potentiality of the $P$. longifolia leaves was investigated against a few clinically isolated as well as standard microbial cultures.

\section{Materials and Methods}

\subsection{Plant Material}

Polyalthia longifolia (Sonn.) Thw. (Annonaceae) leaves were collected in May, 2004 from Rajkot in the State of Gujarat (Western India) and identified by comparison with specimens (PSN 4) available at the Herbarium of the Department of Biosciences, Saurashtra University, Rajkot, Gujarat, India.

\subsection{Extraction}

Leaves of $P$. longifolia were collected, air dried and then powdered in a homogenizer and $10 \mathrm{gm}$ was used for different solvent extractions (Methanol, Acetone, N, $\mathrm{N}$-dimethylformamide); the sample was extracted in solvent kept on a rotary shaker overnight, and then the filtrate was collected and centrifuged at $5000 \mathrm{rpm}$. The solvent was then evaporated to dryness under reduced pressure and the extracted compound left was used for the antimicrobial assay. The percentage yield of 1 , 4-dioxan, methanol and acetone extracts were 20.56, 29.30 and 13.52 respectively.

Microorganisms Studied 91 clinically important microbial strains which included 23 gram positive, 56 gram 
negative and 15 fungal strains were studied for the antimicrobial activity. These strains included both clinical isolates as well as identified strains. The details of the microorganisms used are shown in Table 1.

Table 1. List of bacterial and fungal strains studied for antimicrobial assay.

\begin{tabular}{|c|c|c|}
\hline Sr. & Strain & Specimen \\
\hline & Gram Positive bacteria & \\
\hline 1 & Staphylococcus spps. [10] & Sputum \\
\hline 2 & Staphylococcus aureus [11] & Pus \\
\hline 3 & Staphylococcus aureus [13] & Urine \\
\hline 4 & Staphylococcus aureus [23] & Pus \\
\hline 5 & Staphylococcus spps. [26] & Pus \\
\hline 6 & Staphylococcus aureus [34] & Sputum \\
\hline 7 & Staphylococcus aureus [35] & Tracheal \\
\hline 8 & Staphylococcus aureus [36] & Tracheal \\
\hline 9 & Staphylococcus spps. & Sputum \\
\hline 10 & Staphylococcus aureus [47] & Ear swab \\
\hline 11 & Staphylococcus aureus [48] & Sputum \\
\hline 12 & Staphylococcus aureus [55] & Pus \\
\hline 13 & Staphylococcus aureus [56] & Pus \\
\hline 14 & Staphylococcus aureus ATCC 25923 & - \\
\hline 15 & Staphylococcus epidermidis ATCC 12228 & - \\
\hline 16 & Staphylococcus subfava NCIM 2178 & - \\
\hline 17 & Bacillus cereus ATCC 11778 & - \\
\hline 18 & Bacillus subtilis ATCC 6633 & - \\
\hline 19 & Bacillus megaterium ATCC 9885 & - \\
\hline \multirow[t]{2}{*}{20} & Micrococcus flavus ATCC 10240 & - \\
\hline & Gram negative bacteria & \\
\hline 21 & Pseudomonas spps. [15] & Sputum \\
\hline 22 & Pseudomonas spps. [17] & Pus \\
\hline 23 & Pseudomonas fluorescence [18] & Pus \\
\hline 24 & Pseudomonas spps. [25] & Urine \\
\hline 25 & Pseudomonas spps. [27] & Pus \\
\hline 26 & Pseudomonas aeruginosa [30] & Sputum \\
\hline 27 & Pseudomonas spps. [37] & Tracheal \\
\hline 28 & Pseudomonas aeruginosa [38] & Pus \\
\hline 29 & Pseudomonas spps. [39] & Wound swab \\
\hline 30 & Pseudomonas fluorescence [40] & Tracheal \\
\hline 31 & Pseudomonas spps. [42] & Pus \\
\hline
\end{tabular}

\begin{tabular}{|c|c|c|}
\hline 32 & Pseudomonas spps. [43] & Pus \\
\hline 33 & Pseudomonas spps. [46] & Sputum \\
\hline 34 & Pseudomonas spps. [49] & Sputum \\
\hline 35 & Pseudomonas spps. [50] & Tracheal secretion \\
\hline 36 & Pseudomonas fluorescence [59] & Urine \\
\hline 37 & Pseudomonas aeruginosa ATCC 27853 & - \\
\hline 38 & Pseudomonas testosteroni NCIM 5098 & - \\
\hline 39 & $\begin{array}{l}\text { Pseudomonas pseudoalcaligenes ATCC } \\
17440\end{array}$ & - \\
\hline 40 & E.coli $[14]$ & Pus \\
\hline 41 & E.coli $[16]$ & Urine \\
\hline 42 & E.coli $[21]$ & Urine \\
\hline 43 & E.coli $[22]$ & Urine \\
\hline 44 & E.coli $[24]$ & Urine \\
\hline 45 & E.coli $[28]$ & Pus \\
\hline 46 & E.coli $[31]$ & Urine \\
\hline 47 & E.coli $[32]$ & Stool \\
\hline 48 & E.coli $[33]$ & Pus \\
\hline 49 & E.coli $[41]$ & Urine \\
\hline 50 & E.coli $[45]$ & Pus \\
\hline 51 & E. coli $[51]$ & Urine \\
\hline 52 & E. coli $[58]$ & Vaginal swab \\
\hline 53 & E. coli $[60]$ & Urine \\
\hline 54 & E. coli $[61]$ & Blood \\
\hline 55 & E. coli ATCC 25922 & - \\
\hline 56 & Enterobacter spps. [1] & Tracheal \\
\hline 57 & Enterobacter spps. [8] & Tracheal \\
\hline 58 & Enterobacter aerogenes ATCC 13048 & - \\
\hline 59 & Klebsiella spps [6] & Urine \\
\hline 60 & Klebsiella spps [19] & Sputum \\
\hline 61 & Klebsiella aerogenes [52] & Pus \\
\hline 62 & Klebsiella spps. [54] & Urine \\
\hline 63 & Klebsiella aerogenes [57] & Urine \\
\hline 64 & Klebsiella pneumoniae NCIM 2719 & - \\
\hline 65 & Proteus mirabilis [4] & Wound swab \\
\hline 66 & Proteus spps. [53] & Pus \\
\hline 67 & Proteus mirabilis NCIM 2241 & - \\
\hline 68 & Proteus vulgaris NCTC 8313 & - \\
\hline 69 & Proteus morganii NCIM 2040 & - \\
\hline 70 & Providencia rettgeri [5] & Pus \\
\hline 71 & Citrobacter spps. [20] & Pus \\
\hline
\end{tabular}




\begin{tabular}{ll}
\hline 72 Citrobacter freundii [29] & Pus \\
73 Citrobacter freundii ATCC 10787 & - \\
74 Alcaligenes fecalis ATCC 8750 & - \\
75 Salmonella typhimurium ATCC 23564 & - \\
Fungus & \\
76 Candida albicans [1] & Urine \\
77 Candida albicans [2] & Sputum \\
78 Candida spps. [3] & Sputum \\
79 Candida spps. [4] & Sputum \\
80 Candida spps. [5] & Urine \\
81 Candida albicans ATCC 2091 & - \\
82 Candida albicans ATCC 18804 & - \\
83 Candida glabrata NCIM 3448 & - \\
84 Candida tropicalis ATCC 4563 & - \\
85 Candida apicola NCIM 3367 & - \\
86 Cryptococcus neoformans ATCC 34664 & - \\
87 Cryptococcus luteolus ATCC 32044 & - \\
88 Trichosporan beigelii NCIM 3404 & - \\
89 Aspergillus flavus NCIM 538 & - \\
90 Aspergillus candidus NCIM 883 & - \\
91 Aspergillus niger ATCC 6275 & - \\
\hline &
\end{tabular}

\subsection{Preparation of Samples}

Methanol, acetone and 1,4-dioxan extracts were dissolved in $100 \%$ DMSO at a concentration of $25 \mathrm{mg} / \mathrm{ml}$ and $12.5 \mathrm{mg} / \mathrm{ml}$ and were used as working stocks respectively. Sterile discs (Hi-media Labs) were impregnated with $20 \mu \mathrm{l}$ of the stock solution. Gentamicin (10 $\mu \mathrm{g} / \mathrm{disc})$ and Piperacillin $(100 \mu \mathrm{g} /$ disc) for bacteria; nystatin (100 units/disc) and flucanazole (10 $\mu \mathrm{g} / \mathrm{disc})$ (Himedia Labs) for fungus were used as positive control and pure DMSO was used as a negative control.

\subsection{Antimicrobial Study}

Antimicrobial activity was performed by agar disc diffusion method [8,9]. The bacterial strains were grown in nutrient broth while fungal strains were grown in MGYP (Malt glucose yeast peptone) broth. Mueller Hinton agar no. 2 was the media used to study the antibacterial susceptibility while Sabroaud agar was used to study the antifungal susceptibility test. The cultures were grown for 24 hours, and the turbidity of the culture was maintained according to the 0.5 MacFarland standards. The inoculum's size was $1 \times 10^{8}$ cells $/ \mathrm{ml}$.

\subsection{Agar Disc Diffusion}

The media (Mueller Hinton Agar No.2 and MRS media) and the test bacterial cultures were poured into Petri dishes (Hi-Media). The test strain $(200 \mu \mathrm{l})$ was inoculated into the media (inoculums size $10^{8}$ cells $/ \mathrm{ml}$ ) when the temperature reached $40-42^{\circ} \mathrm{C}$. The test compound $(20$ $\mu \mathrm{l})$ was impregnated in to sterile discs $(7 \mathrm{~mm})$ (Hi-Media) and was then allowed to dry. The disc was then introduced into medium with the bacteria. The plates were incubated overnight at $37^{\circ} \mathrm{C}$ for bacterial strains and $28^{\circ} \mathrm{C}$ for fungal strains. The experiment was performed under strictly aseptic conditions. Microbial growth was determined by measuring the diameter of the zone of inhibition. The experiment was performed in triplicates and the mean values of the result are shown in Table 2 .

\section{Results and Discussion}

Herbal medicine in developing countries is commonly used for the traditional treatment of health problems [10]. In recent years multiple drug resistance in human pathogenic microorganisms has developed due to the indiscriminate use of commercial antimicrobial drugs commonly used in the treatment of infectious diseases, making it a global growing problem [11-13]. In addition to this problem antibiotics are sometimes associated with adverse effects on host including hypersensitivity, immune suppression and allergic reactions [14]. Therefore there is a need to develop alternative antimicrobial drugs for the treatment of infections obtained from various sources such as medicinal plants $[15,16]$. In the present study, $P$. longifolia leaf extracts extracted in 1, 4-dioxan (PDE), methanol (PME) and acetone extracts (PAE) were investigated at two different concentrations for their antimicrobial potentiality against 91 clinically important microbial strains. All the three extracts (PDE, PME and $\mathrm{PAE}$ at $500 \mu \mathrm{g} /$ disc concentration) were active against $95 \%$ of the total gram positive bacterial strains studied. PDE was active against $18.18 \%$ of the total gram negative bacterial strains studied (active against 21\% of Pseudomonas spps., 33.3\% of Enterobacter spps., 16\% of Klebsiella spps., $33.3 \%$ of Proteus spps. and $66.6 \%$ of Citrobacter spps.). PME and PAE were active against $12.72 \%$ of the total gram negative bacterial strains studied. $P$. aeruginosa is most common pathogen of immuno-compromised individuals [17]. Infections caused by Pseudomonas spps. are among the most difficult to treat with conventional antibiotics. Both PME and PAE were active against $5.26 \%$ of the Pseudomonas spps. and $66.6 \%$ of Enterobacter spps. PME was active against $33.3 \%$ of Klebsiella spps. and Proteus spps., while PAE was active against $66.6 \%$ of Klebsiella spps. and Proteus spps. studied. Salmonellosis is an important public 
Table 2. Antimicrobial activity of Polyalthia longifolia against 91 clinically important microbial strains (inhibition zone in $\mathbf{m m})$.

\begin{tabular}{|c|c|c|c|c|c|c|c|c|c|c|c|c|}
\hline \multirow{2}{*}{$\begin{array}{l}\text { Sr. } \\
\text { No. }\end{array}$} & \multirow{2}{*}{ Strain } & \multirow{2}{*}{$\begin{array}{c}\text { Control } \\
\text { DMSO }\end{array}$} & \multicolumn{6}{|c|}{ Extracts } & \multicolumn{4}{|c|}{ Antibiotics } \\
\hline & & & PDE-500 & PME-500 & PAE-500 & PDE-250 & $\begin{array}{l}\text { PME- } \\
250\end{array}$ & $\begin{array}{l}\text { PAE- } \\
250\end{array}$ & G & $\mathbf{P c}$ & $\mathbf{F u}$ & Ns \\
\hline 1 & $\begin{array}{l}\text { Gram Positive } \\
\text { bacteria } \\
\text { Staphylococcus } \\
\text { spps. }[10]\end{array}$ & - & $\begin{array}{l}15 \pm \\
0.58\end{array}$ & $\begin{array}{c}12.67 \pm \\
0.33\end{array}$ & $\begin{array}{l}10 \pm \\
0.58\end{array}$ & $\begin{array}{l}10 \pm \\
0.58\end{array}$ & $\begin{array}{l}11 \pm \\
0.58\end{array}$ & $\begin{array}{c}14.67 \pm \\
0.88\end{array}$ & - & - & NT & NT \\
\hline 2 & $\begin{array}{l}\text { Staphylococcus } \\
\text { aureus [11] }\end{array}$ & - & $\begin{array}{l}13 \pm \\
0.58\end{array}$ & $\begin{array}{l}12 \pm \\
0.58\end{array}$ & $\begin{array}{l}10 \pm \\
0.58\end{array}$ & $\begin{array}{l}11 \pm \\
0.58\end{array}$ & $\begin{array}{l}12 \pm \\
1.15\end{array}$ & $\begin{array}{l}12 \pm \\
0.58\end{array}$ & $\begin{array}{l}18.67 \pm \\
0.33\end{array}$ & $\begin{array}{c}17.33 \\
\pm \\
0.33\end{array}$ & NT & NT \\
\hline 3 & $\begin{array}{l}\text { Staphylococcus } \\
\text { aureus [13] }\end{array}$ & - & $\begin{array}{l}12 \pm \\
1.15\end{array}$ & $\begin{array}{l}12 \pm \\
2.31\end{array}$ & $\begin{array}{c}9 \pm \\
0.58\end{array}$ & - & - & - & - & - & NT & NT \\
\hline 4 & $\begin{array}{l}\text { Staphylococcus } \\
\text { aureus [23] }\end{array}$ & - & $\begin{array}{l}11 \pm \\
0.58\end{array}$ & $\begin{array}{l}12 \pm \\
1.73\end{array}$ & $\begin{array}{c}9 \pm \\
0.58\end{array}$ & $\begin{array}{l}12 \pm \\
1.73\end{array}$ & $\begin{array}{l}9 \pm \\
1.15\end{array}$ & - & - & - & NT & NT \\
\hline 5 & $\begin{array}{l}\text { Staphylococcus } \\
\text { spps [26] }\end{array}$ & - & $\begin{array}{c}16.5 \pm \\
0.28\end{array}$ & $\begin{array}{l}11 \pm \\
0.58\end{array}$ & $\begin{array}{l}13 \pm \\
0.58\end{array}$ & $\begin{array}{l}15 \pm \\
0.58\end{array}$ & $\begin{array}{l}13 \pm \\
1.73\end{array}$ & $\begin{array}{l}14 \pm \\
1.73\end{array}$ & - & - & NT & NT \\
\hline 6 & $\begin{array}{l}\text { Staphylococcus } \\
\text { aureus }[34]\end{array}$ & - & $\begin{array}{c}15.5 \pm \\
0.28\end{array}$ & $\begin{array}{c}9 \pm \\
0.58\end{array}$ & $\begin{array}{l}13 \pm \\
0.58\end{array}$ & $\begin{array}{l}14 \pm \\
0.58\end{array}$ & $\begin{array}{c}9 \pm \\
0.58\end{array}$ & $\begin{array}{l}10 \pm \\
1.15\end{array}$ & - & - & NT & NT \\
\hline 7 & $\begin{array}{l}\text { Staphylococcus } \\
\text { aureus [35] }\end{array}$ & - & $\begin{array}{l}22 \pm \\
0.28\end{array}$ & $\begin{array}{l}12 \pm \\
0.28\end{array}$ & $\begin{array}{l}14 \pm \\
0.58\end{array}$ & $\begin{array}{l}17 \pm \\
0.58\end{array}$ & $\begin{array}{c}8 \pm \\
0.58\end{array}$ & $\begin{array}{l}11 \pm \\
0.58\end{array}$ & - & - & NT & NT \\
\hline 8 & $\begin{array}{l}\text { Staphylococcus } \\
\text { aureus [36] }\end{array}$ & - & $\begin{array}{l}13 \pm \\
0.58\end{array}$ & $\begin{array}{l}9.67 \pm \\
0.33\end{array}$ & $\begin{array}{l}13 \pm \\
0.58\end{array}$ & $\begin{array}{c}12.67 \pm \\
0.88\end{array}$ & - & $\begin{array}{c}8.67 \pm \\
0.88\end{array}$ & - & - & NT & NT \\
\hline 9 & $\begin{array}{l}\text { Staphylococcus } \\
\text { spps [44] }\end{array}$ & - & $\begin{array}{l}13 \pm \\
0.58\end{array}$ & $\begin{array}{c}10.33 \pm \\
0.33\end{array}$ & $\begin{array}{c}12.33 \pm \\
0.33\end{array}$ & $\begin{array}{c}18 \\
0.58\end{array}$ & $\begin{array}{l}11 \pm \\
0.58\end{array}$ & $\begin{array}{c}10.67 \\
\pm \\
0.66\end{array}$ & $\begin{array}{c}14.67 \pm \\
0.33\end{array}$ & - & NT & NT \\
\hline 10 & $\begin{array}{l}\text { Staphylococcus } \\
\text { aureus [47] }\end{array}$ & - & $\begin{array}{l}12 \pm \\
3.21\end{array}$ & $\begin{array}{c}10.67 \pm \\
2.03\end{array}$ & $\begin{array}{l}11 \pm \\
2.31\end{array}$ & $\begin{array}{c}9 \pm \\
1.15\end{array}$ & $\begin{array}{c}8.67 \pm \\
0.88\end{array}$ & $\begin{array}{l}12 \pm \\
2.89\end{array}$ & - & - & NT & NT \\
\hline 11 & $\begin{array}{l}\text { Staphylococcus } \\
\text { aureus [48] }\end{array}$ & - & - & - & - & - & - & - & $\begin{array}{c}20.67 \pm \\
0.33\end{array}$ & - & NT & NT \\
\hline 12 & $\begin{array}{l}\text { Staphylococcus } \\
\text { aureus [55] }\end{array}$ & - & $\begin{array}{c}13.67 \pm \\
0.33\end{array}$ & $\begin{array}{l}12.67 \pm \\
0.33\end{array}$ & $\begin{array}{c}13.67 \pm \\
0.33\end{array}$ & $\begin{array}{c}11.67 \pm \\
0.33\end{array}$ & - & - & - & - & NT & NT \\
\hline 13 & $\begin{array}{l}\text { Staphylococcus } \\
\text { aureus }[56]\end{array}$ & - & $\begin{array}{c}15.67 \pm \\
0.33\end{array}$ & $\begin{array}{l}10 \pm \\
1.53\end{array}$ & $\begin{array}{c}11.67 \pm \\
0.88\end{array}$ & $\begin{array}{c}12.33 \pm \\
0.33\end{array}$ & $\begin{array}{l}10.33 \\
\pm \\
1.76\end{array}$ & $\begin{array}{c}12.67 \\
\pm \\
0.33\end{array}$ & $\begin{array}{c}10.33 \pm \\
0.33\end{array}$ & - & NT & NT \\
\hline 14 & $\begin{array}{l}\text { Staphylococcus } \\
\text { aureus ATCC } \\
25923\end{array}$ & - & $\begin{array}{l}13 \pm \\
0.58\end{array}$ & $\begin{array}{c}8 \pm \\
0.58\end{array}$ & $\begin{array}{c}9 \pm \\
0.58\end{array}$ & $\begin{array}{c}14.33 \pm \\
0.88\end{array}$ & $\begin{array}{c}9.5 \pm \\
0.28\end{array}$ & $\begin{array}{l}9 \pm \\
0.58\end{array}$ & - & - & NT & NT \\
\hline 15 & $\begin{array}{l}\text { Staphylococcus } \\
\text { epidermidis ATCC } \\
12228\end{array}$ & - & $\begin{array}{c}14.5 \pm \\
2.60\end{array}$ & $\begin{array}{l}16 \pm \\
2.69\end{array}$ & $13 \pm 0.58$ & $\begin{array}{c}13.5 \pm \\
0.87\end{array}$ & $\begin{array}{l}13 \pm \\
0.57\end{array}$ & $\begin{array}{l}12 \pm \\
1.73\end{array}$ & - & - & NT & NT \\
\hline 16 & $\begin{array}{l}\text { Staphylococcus } \\
\text { subfava NCIM } \\
2178\end{array}$ & - & $\begin{array}{c}10.5 \pm \\
0.29\end{array}$ & $\begin{array}{c}11.5 \pm \\
1.44\end{array}$ & $\begin{array}{c}12.5 \pm \\
0.28\end{array}$ & $13 \pm 2.31$ & $\begin{array}{c}9.5 \pm \\
0.28\end{array}$ & $\begin{array}{c}9.5 \pm \\
0.28\end{array}$ & - & $\begin{array}{l}20.17 \\
\pm \\
0.44\end{array}$ & NT & NT \\
\hline 17 & $\begin{array}{l}\text { Bacillus cereus } \\
\text { ATCC } 11778\end{array}$ & - & $\begin{array}{c}29.5 \pm \\
0.28\end{array}$ & $\begin{array}{c}21.5 \pm \\
0.28\end{array}$ & $\begin{array}{c}25 . \pm \\
0.58\end{array}$ & $25 \pm 2.31$ & $\begin{array}{l}21 \pm \\
0.58\end{array}$ & $\begin{array}{l}25 \pm \\
0.58\end{array}$ & $\begin{array}{l}20.17 \\
\pm 0.16\end{array}$ & $\begin{array}{c}18.83 \\
\pm \\
0.16\end{array}$ & NT & NT \\
\hline 18 & $\begin{array}{l}\text { Bacillus subtilis } \\
\text { ATCC } 6633\end{array}$ & - & $\begin{array}{c}26.5 \pm \\
1.44\end{array}$ & $\begin{array}{c}21.5 \pm \\
1.44\end{array}$ & $\begin{array}{c}23.5 \pm \\
0.28\end{array}$ & $25 \pm 0.58$ & $\begin{array}{l}21 \pm \\
0.58\end{array}$ & $\begin{array}{l}21 \pm \\
0.58\end{array}$ & $\begin{array}{c}18.33 \\
\pm 0.33\end{array}$ & $\begin{array}{c}17.83 \\
\pm \\
0.93\end{array}$ & NT & NT \\
\hline 19 & $\begin{array}{l}\text { Bacillus } \\
\text { megaterium ATCC } \\
9885\end{array}$ & - & $14 \pm 0.58$ & $\begin{array}{c}10.5 \pm \\
0.28\end{array}$ & $\begin{array}{c}12.5 \pm \\
0.28\end{array}$ & $13 \pm 0.58$ & $\begin{array}{l}11 \pm \\
0.58\end{array}$ & $\begin{array}{c}10.5 \pm \\
0.28\end{array}$ & - & - & NT & NT \\
\hline 20 & $\begin{array}{l}\text { Micrococcus } \\
\text { flavus ATCC } \\
10240\end{array}$ & - & $\begin{array}{c}12.5 \pm \\
0.28\end{array}$ & $\begin{array}{c}10.5 \pm \\
0.28\end{array}$ & $11 \pm 2.31$ & $\begin{array}{c}11.5 \pm \\
0.28\end{array}$ & $\begin{array}{c}9 \pm \\
0.58\end{array}$ & $\begin{array}{c}9 \pm \\
0.58\end{array}$ & $\begin{array}{l}27.67 \\
\pm 0.33\end{array}$ & $\begin{array}{c}12.67 \\
\pm \\
0.33\end{array}$ & NT & NT \\
\hline & $\begin{array}{l}\text { Gram negative } \\
\text { bacteria }\end{array}$ & & & & & & & & & & NT & NT \\
\hline 21 & $\begin{array}{l}\text { Pseudomonas } \\
\text { spps. [15] }\end{array}$ & - & - & - & - & - & - & - & $\begin{array}{l}14 \pm \\
0.58\end{array}$ & - & NT & NT \\
\hline 22 & $\begin{array}{l}\text { Pseudomonas } \\
\text { spps. [17] }\end{array}$ & - & $\begin{array}{c}8 \pm \\
0.58\end{array}$ & - & - & - & - & $\begin{array}{l}12 \pm \\
2.89\end{array}$ & - & - & NT & NT \\
\hline 23 & $\begin{array}{l}\text { Pseudomonas } \\
\text { fluorescence }[18]\end{array}$ & - & $\begin{array}{c}8 \pm \\
0.58\end{array}$ & - & - & - & - & $\begin{array}{l}12 \pm \\
2.89\end{array}$ & - & - & NT & NT \\
\hline 24 & $\begin{array}{l}\text { Pseudomonas } \\
\text { spps. [25] }\end{array}$ & - & - & - & - & - & - & - & - & - & NT & NT \\
\hline
\end{tabular}




\begin{tabular}{|c|c|c|c|c|c|c|c|c|c|c|c|c|}
\hline 25 & $\begin{array}{l}\text { Pseudomonas } \\
\text { spps. [27] }\end{array}$ & - & - & - & - & - & - & - & - & - & NT & NT \\
\hline 26 & $\begin{array}{l}\text { Pseudomonas } \\
\text { aeruginosa }[30]\end{array}$ & - & -- & - & - & - & - & - & $\begin{array}{c}16.67 \pm \\
0.67\end{array}$ & - & NT & NT \\
\hline 27 & $\begin{array}{l}\text { Pseudomonas } \\
\text { spps. [37] }\end{array}$ & - & -- & - & - & - & - & - & - & -- & NT & NT \\
\hline 28 & $\begin{array}{l}\text { Pseudomonas } \\
\text { aeruginosa }[38]\end{array}$ & - & - & - & - & - & - & - & $\begin{array}{c}19.67 \pm \\
0.33\end{array}$ & - & NT & NT \\
\hline 29 & $\begin{array}{l}\text { Pseudomonas } \\
\text { spps. [39] }\end{array}$ & - & - & - & - & - & - & - & - & - & NT & NT \\
\hline 30 & $\begin{array}{l}\text { Pseudomonas } \\
\text { fluorescence [40] }\end{array}$ & - & - & - & - & - & - & - & - & - & NT & NT \\
\hline 31 & $\begin{array}{l}\text { Pseudomonas } \\
\text { spps. [42] }\end{array}$ & - & - & - & - & - & - & - & - & - & NT & NT \\
\hline 32 & $\begin{array}{l}\text { Pseudomonas } \\
\text { spps. [43] }\end{array}$ & - & - & - & - & - & - & - & - & - & NT & NT \\
\hline 33 & $\begin{array}{l}\text { Pseudomonas } \\
\text { spps. [46] }\end{array}$ & - & - & - & - & - & - & - & - & - & NT & NT \\
\hline 34 & $\begin{array}{l}\text { Pseudomonas } \\
\text { spps. [49] }\end{array}$ & - & $\begin{array}{c}8 \pm \\
0.58\end{array}$ & - & - & - & - & $\begin{array}{c}8 \pm \\
0.58\end{array}$ & $\begin{array}{l}20 \pm \\
0.58\end{array}$ & - & NT & NT \\
\hline 35 & $\begin{array}{l}\text { Pseudomonas } \\
\text { spps. [50] }\end{array}$ & - & - & - & - & - & - & - & - & - & NT & NT \\
\hline 36 & $\begin{array}{l}\text { Pseuodmonas } \\
\text { fluorescence [59] }\end{array}$ & - & - & - & - & - & - & - & - & - & NT & NT \\
\hline 37 & $\begin{array}{l}\text { Pseudomonas } \\
\text { aeruginosa ATCC } \\
27853\end{array}$ & - & - & - & - & - & - & - & $\begin{array}{l}17 \pm \\
1.15\end{array}$ & $\begin{array}{c}12.33 \\
\pm \\
0.66\end{array}$ & NT & NT \\
\hline 38 & $\begin{array}{l}\text { Pseudomonas } \\
\text { testosteroni NCIM } \\
5098\end{array}$ & - & - & - & - & - & - & - & $\begin{array}{l}22.33 \\
\pm 0.66\end{array}$ & - & NT & NT \\
\hline 39 & $\begin{array}{l}\text { Pseudomonas } \\
\text { pseudoalcaligenes } \\
\text { ATCC } 17440\end{array}$ & - & $\begin{array}{c}8.5 \pm \\
0.86\end{array}$ & $14 \pm 1.73$ & $\begin{array}{c}10.5 \pm \\
0.86\end{array}$ & - & - & - & $\begin{array}{l}19.33 \\
\pm 0.6\end{array}$ & - & NT & NT \\
\hline 40 & E.coli $[14]$ & - & - & - & - & - & - & - & - & - & NT & NT \\
\hline 41 & E.coli $[16]$ & - & - & - & - & - & - & - & - & - & NT & NT \\
\hline 42 & E.coli $[21]$ & - & - & - & - & - & - & - & - & - & NT & NT \\
\hline 43 & E.coli $[22]$ & - & - & - & - & - & - & - & - & - & NT & NT \\
\hline 44 & E.coli $[24]$ & - & - & - & - & - & - & - & - & - & NT & NT \\
\hline 45 & E.coli $[28]$ & - & & & & & & $\begin{array}{l}17 \pm \\
0.33\end{array}$ & & & NT & NT \\
\hline 46 & E.coli [31] & - & - & - & - & - & - & - & - & - & NT & NT \\
\hline 47 & E.coli [32] & - & - & - & - & - & - & - & $\begin{array}{l}21 \pm \\
0.58\end{array}$ & - & NT & NT \\
\hline 48 & E.coli $[33]$ & - & - & - & - & - & - & - & - & - & NT & NT \\
\hline 49 & E.coli $[41]$ & - & - & - & - & - & - & - & $\begin{array}{c}18.67 \pm \\
0.33\end{array}$ & - & NT & NT \\
\hline 50 & E.coli [45] & - & - & - & - & - & - & - & - & - & NT & NT \\
\hline 51 & E. coli [51] & - & - & - & - & - & - & - & $\begin{array}{c}20.33 \pm \\
0.33\end{array}$ & - & NT & NT \\
\hline 52 & E. coli $[58]$ & - & - & - & - & - & - & - & - & - & NT & NT \\
\hline 53 & E. coli $[60]$ & - & - & - & - & - & - & - & - & - & NT & NT \\
\hline 54 & E. coli $[61]$ & - & - & - & - & - & - & - & - & - & NT & NT \\
\hline 55 & $\begin{array}{l}\text { E. coli ATCC } \\
25922\end{array}$ & - & - & - & - & - & - & - & $\begin{array}{c}17.83 \\
\pm \\
0.16\end{array}$ & $\begin{array}{c}14.5 \\
\pm \\
0.50\end{array}$ & NT & NT \\
\hline 56 & $\begin{array}{l}\text { Enterobacter spps. } \\
{[1]}\end{array}$ & - & - & - & - & - & - & - & - & - & NT & NT \\
\hline 57 & $\begin{array}{l}\text { Enterobacter spps. } \\
{[8]}\end{array}$ & - & $\begin{array}{l}15 \pm \\
0.58\end{array}$ & $\begin{array}{l}12 \pm \\
0.58\end{array}$ & $\begin{array}{c}14.33 \pm \\
1.20\end{array}$ & $\begin{array}{l}13 \pm \\
0.58\end{array}$ & $\begin{array}{c}12.33 \\
\pm \\
0.88\end{array}$ & $\begin{array}{l}12 \pm \\
1.15\end{array}$ & $\begin{array}{c}19.67 \pm \\
0.88\end{array}$ & - & NT & NT \\
\hline 58 & $\begin{array}{l}\text { Enterobacter } \\
\text { aerogenes ATCC } \\
13048\end{array}$ & - & - & $\begin{array}{l}8.5 \pm \\
0.86\end{array}$ & $\begin{array}{l}15 \pm \\
0.58\end{array}$ & - & - & - & - & - & NT & NT \\
\hline 59 & Klebsiella spps [6] & - & - & - & - & - & - & - & $\begin{array}{l}22 \pm \\
0.58\end{array}$ & - & NT & NT \\
\hline 60 & $\begin{array}{l}\text { Klebsiella spps } \\
{[19]}\end{array}$ & - & - & - & - & - & - & - & - & - & NT & NT \\
\hline 61 & $\begin{array}{l}\text { Klebsiella aero- } \\
\text { genes }[52]\end{array}$ & - & - & - & $\begin{array}{c}8 \pm \\
0.58 \\
\end{array}$ & $\begin{array}{l}13 \pm \\
1.73 \\
\end{array}$ & $\begin{array}{l}11 \pm \\
2.08\end{array}$ & - & - & - & NT & NT \\
\hline
\end{tabular}




\begin{tabular}{|c|c|c|c|c|c|c|c|c|c|c|c|c|}
\hline 62 & $\begin{array}{l}\text { Klebsiella spps. } \\
\text { [54] }\end{array}$ & - & - & - & - & - & - & - & - & - & NT & NT \\
\hline 63 & $\begin{array}{l}\text { Klebsiella aero- } \\
\text { genes [57] }\end{array}$ & - & - & - & - & - & - & - & - & - & NT & NT \\
\hline 64 & $\begin{array}{l}\text { Klebsiella pneu- } \\
\text { moniae NCIM } \\
2719\end{array}$ & - & $\begin{array}{l}12 \pm \\
0.58\end{array}$ & $\begin{array}{l}12 \pm \\
0.58\end{array}$ & $\begin{array}{c}10.5 \pm \\
0.28\end{array}$ & $\begin{array}{c}10.5 \pm \\
0.86\end{array}$ & $\begin{array}{c}10.5 \pm \\
0.86\end{array}$ & $\begin{array}{l}11 \pm \\
0.58\end{array}$ & - & $\begin{array}{c}24.67 \\
\pm \\
0.33\end{array}$ & NT & NT \\
\hline 65 & $\begin{array}{l}\text { Proteus mirabilis } \\
\text { [4] }\end{array}$ & - & - & - & - & - & - & - & - & $\begin{array}{l}14 \pm \\
0.58\end{array}$ & NT & NT \\
\hline 66 & Proteus spps. [53] & - & - & - & - & - & -- & - & - & - & NT & NT \\
\hline 67 & $\begin{array}{l}\text { Proteus mirabilis } \\
\text { NCIM } 2241\end{array}$ & - & $\begin{array}{c}10.5 \pm \\
0.86\end{array}$ & $\begin{array}{c}10.5 \pm \\
0.28\end{array}$ & $\begin{array}{l}9.5 \pm \\
0.86\end{array}$ & - & - & - & $\begin{array}{r}18.67 \\
\pm 0.33\end{array}$ & - & NT & NT \\
\hline 68 & $\begin{array}{l}\text { Proteus vulgaris } \\
\text { NCTC } 8313\end{array}$ & - & - & $9 \pm 1.15$ & - & - & - & - & $\begin{array}{l}18 \pm \\
1.00\end{array}$ & - & NT & NT \\
\hline 69 & $\begin{array}{l}\text { Proteus morganii } \\
\text { NCIM } 2040\end{array}$ & - & $9 \pm 0.58$ & - & - & $8 \pm 0.58$ & - & - & - & - & NT & NT \\
\hline 70 & $\begin{array}{l}\text { Providencia rett- } \\
\text { geri }[5]\end{array}$ & - & - & - & - & - & - & - & - & - & NT & NT \\
\hline 71 & $\begin{array}{l}\text { Citrobactor spps } \\
{[20]}\end{array}$ & - & $\begin{array}{c}8 \pm \\
0.58\end{array}$ & $\begin{array}{c}8 \pm \\
0.58\end{array}$ & $\begin{array}{c}8 \pm \\
0.58\end{array}$ & - & - & - & - & - & NT & NT \\
\hline 72 & $\begin{array}{l}\text { Citrobactor } \\
\text { freundii [29] }\end{array}$ & - & - & - & - & - & - & - & $\begin{array}{c}12.33 \pm \\
0.33\end{array}$ & - & NT & NT \\
\hline 73 & $\begin{array}{l}\text { Citrobactor } \\
\text { freundii ATCC } \\
10787\end{array}$ & - & $11 \pm 0.58$ & - & - & $\begin{array}{c}11.5 \pm \\
0.28\end{array}$ & $\begin{array}{l}10 \pm \\
0.58\end{array}$ & $\begin{array}{c}9.5 \pm \\
0.28\end{array}$ & - & - & NT & NT \\
\hline 74 & $\begin{array}{l}\text { Alcaligenes fecalis } \\
\text { ATCC } 8750\end{array}$ & - & - & - & - & - & - & - & $\begin{array}{l}18.33 \\
\pm 0.66\end{array}$ & - & NT & NT \\
\hline 75 & $\begin{array}{l}\text { Salmonella ty- } \\
\text { phimurium ATCC } \\
23564 \\
\text { Fungus }\end{array}$ & - & - & - & - & - & - & - & $\begin{array}{c}18.5 \pm \\
0.28\end{array}$ & - & NT & NT \\
\hline 76 & $\begin{array}{l}\text { Candida albicans } \\
\text { [1] }\end{array}$ & - & $\begin{array}{l}7.5 \pm \\
0.29\end{array}$ & $\begin{array}{c}8 \pm \\
0.58\end{array}$ & - & $\begin{array}{l}7.5 \pm \\
0.29\end{array}$ & $\begin{array}{c}10.5 \pm \\
0.29\end{array}$ & $\begin{array}{l}10 \pm \\
0.58\end{array}$ & NT & NT & - & $\begin{array}{c}11.33 \\
\pm \\
0.33\end{array}$ \\
\hline 77 & $\begin{array}{l}\text { Candida albicans } \\
{[2]}\end{array}$ & - & - & - & $\begin{array}{l}10 \pm \\
0.58\end{array}$ & $\begin{array}{c}13.33 \pm \\
0.88\end{array}$ & $\begin{array}{c}9 \pm \\
0.58\end{array}$ & - & NT & NT & - & $\begin{array}{l}18 \pm \\
0.58\end{array}$ \\
\hline 78 & Candida spps. [3] & - & - & - & $\begin{array}{c}9.5 \pm \\
0.29\end{array}$ & $\begin{array}{l}14.33 \pm \\
0.66\end{array}$ & $\begin{array}{c}12.5 \pm \\
0.86\end{array}$ & $\begin{array}{c}8 \pm \\
0.58\end{array}$ & NT & NT & - & $\begin{array}{l}14 \pm \\
0.58\end{array}$ \\
\hline 79 & Candida spps. [4] & - & $\begin{array}{l}11 \pm \\
2.13\end{array}$ & $\begin{array}{c}10.5 \pm \\
2.02\end{array}$ & $\begin{array}{l}11.5 \pm \\
2.06\end{array}$ & $\begin{array}{c}8 \pm \\
0.58\end{array}$ & $\begin{array}{l}8.5 \pm \\
0.29\end{array}$ & $\begin{array}{c}12.5 \pm \\
0.86\end{array}$ & NT & NT & - & $\begin{array}{l}14 \pm \\
0.58\end{array}$ \\
\hline 80 & Candida spps. [5] & - & $\begin{array}{l}7.5 \pm \\
0.29\end{array}$ & $\begin{array}{l}8.5 \pm \\
0.29\end{array}$ & $\begin{array}{c}9.5 \pm \\
0.29\end{array}$ & $\begin{array}{l}7.5 \pm \\
0.29\end{array}$ & - & 0.00 & NT & NT & - & $\begin{array}{l}10 \pm \\
0.58\end{array}$ \\
\hline 81 & $\begin{array}{l}\text { Candida albicans } \\
\text { ATCC } 2091\end{array}$ & - & $\begin{array}{l}11.5 \pm \\
2.60\end{array}$ & $\begin{array}{l}11 \pm \\
2.31\end{array}$ & $\begin{array}{c}8 \pm \\
0.58\end{array}$ & $\begin{array}{l}7.5 \pm \\
0.29\end{array}$ & $\begin{array}{l}7.5 \pm \\
0.29\end{array}$ & $\begin{array}{c}10.5 \pm \\
2.02\end{array}$ & NT & NT & $\begin{array}{l}17.67 \\
\pm \\
0.33\end{array}$ & $\begin{array}{l}13 \pm \\
0.58\end{array}$ \\
\hline 82 & $\begin{array}{l}\text { Candida albicans } \\
\text { ATCC } 18804\end{array}$ & - & $\begin{array}{c}10.5 \pm \\
0.29\end{array}$ & $\begin{array}{c}8 \pm \\
0.58\end{array}$ & - & - & $\begin{array}{l}11 \pm \\
0.58\end{array}$ & $\begin{array}{l}15 \pm \\
1.15\end{array}$ & NT & NT & - & $\begin{array}{c}14.33 \\
\pm \\
0.33\end{array}$ \\
\hline 83 & $\begin{array}{l}\text { Candida glabrata } \\
\text { NCIM } 3448\end{array}$ & - & - & - & - & - & - & - & NT & NT & $\begin{array}{l}39.67 \\
\pm \\
0.88\end{array}$ & $\begin{array}{l}22 \pm \\
0.58\end{array}$ \\
\hline 84 & $\begin{array}{l}\text { Candida tropicalis } \\
\text { ATCC } 4563\end{array}$ & - & - & - & $\begin{array}{l}7.5 \pm \\
0.29\end{array}$ & $\begin{array}{l}11 \pm \\
0.58\end{array}$ & $\begin{array}{l}12 \pm \\
0.58\end{array}$ & $\begin{array}{l}9.5 \pm \\
0.29\end{array}$ & NT & NT & - & $\begin{array}{l}8.33 \\
\pm \\
0.33\end{array}$ \\
\hline 85 & $\begin{array}{l}\text { Candida apicola } \\
\text { NCIM } 3367\end{array}$ & - & $\begin{array}{l}23 \pm \\
3.60\end{array}$ & $\begin{array}{l}26 \pm \\
0.58\end{array}$ & $\begin{array}{l}28 \pm \\
1.15\end{array}$ & $\begin{array}{c}25.33 \pm \\
0.88\end{array}$ & $\begin{array}{l}24 \pm \\
0.58\end{array}$ & $\begin{array}{l}21.66 \\
\pm \\
0.33\end{array}$ & NT & NT & - & $\begin{array}{c}21.33 \\
\pm \\
0.88\end{array}$ \\
\hline 86 & $\begin{array}{l}\text { Cryptococcus } \\
\text { neoformans ATCC } \\
34664\end{array}$ & - & $\begin{array}{l}7.5 \pm \\
0.29\end{array}$ & $\begin{array}{c}8 \pm \\
0.58\end{array}$ & - & - & - & $\begin{array}{l}9.5 \pm \\
1.4\end{array}$ & NT & NT & $\begin{array}{c}21.33 \\
\pm \\
0.33\end{array}$ & $\begin{array}{l}17 \pm \\
0.58\end{array}$ \\
\hline 87 & $\begin{array}{l}\text { Cryptococcus } \\
\text { luteolus ATCC } \\
32044\end{array}$ & - & $\begin{array}{l}14 \pm \\
0.58\end{array}$ & $\begin{array}{c}11.5 \pm \\
0.86\end{array}$ & $\begin{array}{l}11 \pm \\
1.15\end{array}$ & $\begin{array}{c}9.5 \pm \\
1.44\end{array}$ & $\begin{array}{l}8.5 \pm \\
0.86\end{array}$ & $\begin{array}{l}8.5 \pm \\
0.88\end{array}$ & NT & NT & $\begin{array}{l}23.66 \\
\pm \\
0.88\end{array}$ & $\begin{array}{c}17.66 \\
\pm \\
0.88\end{array}$ \\
\hline 88 & $\begin{array}{l}\text { Trichosporan } \\
\text { beigelii NCIM } \\
3404\end{array}$ & - & $\begin{array}{l}12 \pm \\
0.58\end{array}$ & $\begin{array}{l}13 \pm \\
1.73\end{array}$ & $\begin{array}{c}10.5 \pm \\
2.02\end{array}$ & - & - & - & NT & NT & - & - \\
\hline 89 & $\begin{array}{l}\text { Aspergillus flavus } \\
\text { NCIM } 538\end{array}$ & - & - & - & - & $\begin{array}{c}14.67 \pm \\
4.34\end{array}$ & $\begin{array}{l}22 \pm \\
0.58\end{array}$ & $\begin{array}{l}10.33 \\
\pm 2.02\end{array}$ & NT & NT & - & - \\
\hline
\end{tabular}




\begin{tabular}{|c|c|c|c|c|c|c|c|c|c|c|c|c|}
\hline 90 & $\begin{array}{l}\text { Aspergillus can- } \\
\text { didus NCIM } 883\end{array}$ & - & $\begin{array}{c}10.5 \pm \\
0.29\end{array}$ & $\begin{array}{l}9 \pm \\
1.15\end{array}$ & $\begin{array}{l}11 \pm \\
0.58\end{array}$ & - & - & - & NT & NT & - & - \\
\hline 91 & $\begin{array}{l}\text { Aspergillus niger } \\
\text { ATCC } 6275\end{array}$ & - & - & - & - & $\begin{array}{l}11 \pm \\
2.31\end{array}$ & - & - & NT & NT & - & - \\
\hline
\end{tabular}

health problem worldwide. Salmonella infection is primarily associated with gastroenteritis. This illness poses a more serious health risk to sensitive populations in the community such as the elderly, young and the immunocompromised, where hospitalization may be required. All the three extracts were inactive against E. coli, A. fecalis and $S$. typhimurium. Several antimycotic drugs are available at present, its use is limited by a number of factors such as low potency, poor solubility, emergence of resistance strains and drug toxicity. Therefore there is distinct need for the discovery of new, safer and more effective antifungal agents. Candida species have become a common cause of hospital acquired infections and a large number of patients die as a result of invasive Candidal infections [18]. All the three extracts were active against $62.5 \%$ of the total fungal strains studied. The three extracts were active against $A$. candidus while it was inactive against the remaining two moulds (A.flavus and $A$. niger) studied. The details of the results are given elaborately in Table 2. From the results obtained, it seems that the antibacterial action of the extracts is more pronounced on gram positive than on gram negative bacteria and these findings correlate with the observations of previous screenings of medicinal plants for antimicrobial activity, where most of the active plants showed activity against gram positive strains only [1921]. This difference in susceptibility is because of the difference in cell wall structure of gram positive and gram negative organisms. The lipopolysaccharide content of gram negative bacteria makes them resistant to plant extracts while the peptidoglycan layer of gram positive bacteria is not an effective permeability barrier.

\section{Conclusions}

All the extracts of $P$. longifolia exhibited the highest rates of antimicrobial activity against gram positive and fungal strains studied. Therefore, it is concluded that $P$. longifolia extracts should further be studied phytochemically to elucidate the active principle in the leaf, which can be used as a leading antibacterial (specific for gram positive) and antifungal agent.

\section{Acknowledgements}

Financial support from Department of Special assistance (DSA) project, New Delhi and supply of clinical isolates by Micro Care and Spandan Diagnostic Laboratories,
Rajkot are gratefully acknowledged.

\section{References}

[1] C. K. Amadou, "Promoting Alternative Medicine," Africa Health Journal, 1998, Vol. 2, pp. 20-25.

[2] J. D. Hooker and C. B. Clarke, "Flora of British India, Vol. 1," L. Reeve and Co. Ltd., London, 1875, pp. 1-741.

[3] K. Raghunathan and M. K. Mitra, "Pharmacognosy of Indigenous Drugs, Vol. 1," Central Council for Research in Ayurveda and Siddha, New Delhi, 1985, pp. 127-139.

[4] S. K. Chakrabarti and B. Mukherjee, "Search for Anticancer Drug from Indian Medicinal Plants," Indian Journal of Medical Research, Vol. 56, No. 4, 1968, pp. 445-455.

[5] K. Yamaguchi, H. Kinora, S. Natori, Ito, K. Nissbimoio, K. Bando, D. Mizuno and M. Ishignoo, "Screening Tests for Antitumor Activity of Asian Medicinal Herbs I," Yakugaku Zashi, 1964, Vol. 84, pp. 373-377.

[6] K. R. Kirtikar and B. D. Basu, "Indian Medicinal Plants," In: Annonaceae, 2nd Edition, Lalit Mohan Basu, Leader Road, Allahabad, India, Vol. 1, pp. 1993, pp. 72-73.

[7] C. M. Hasan, S. N. Islam and M. Ahsan, "Antibacterial Activity of Stem Bark of Polyalthia longifolia," Dhaka University Studies, Part E, 1988, Vol. 4, pp. 63-66.

[8] A. W. Bauer, W. M. M. Kirby, J. C. Sherries and M. Truck, "Antibiotic Susceptibility Testing By Standard Single Disc Diffusion Method," American Journal of Clinical Pathology, Vol. 45, No. 4, 1966, pp. 426-493.

[9] J. Parekh and S. Chanda, "Antibacterial and Phytochemical Studies on Twelve Species of Indian Medicinal Plants," African Journal of Biomedical Research, Vol. 10, No. 2, 2007, pp. 175-181.

[10] M. J. Martinez, J. Betancourt, N. Alanso-Gonzalea and A. Jauregui, "Screening of Some Cuban Medicinal Plants for Antimicrobial Activity," Journal of Ethnopharmacology, Vol. 52, No. 3, 1996, pp. 171-174.

[11] J. E. Loper, M. D. Henkels, R. G. Roberts, G. G. Grove, M. J. Willett and T. J. Smith, "Evaluation of Streptomycin, Oxytetracycline and Copper Resistance of Erwinia amylavora isolated from pear orchards in Washington State," Plant Disease, Vol. 75, No. 3, 1991, pp. 287-290.

[12] J. Davis, "Inactivation of Antibiotics and Dissemination of Resistance Genes," Science, Vol. 264, No. 5157, 1994, pp. 375-382.

[13] R. F. Service, "Antibiotics That Resist Resistance," Science, Vol. 270, No. 5237, 1995, pp. 724-727.

[14] I. Ahmad, Z. Mehmood and F. Mohammad, "Screening of Some Indian Medicinal Plants for their Antimicrobial Properties," Journal of Ethnopharmacology, Vol. 62, No. 
2, 1998, pp. 183-193.

[15] A. M. Clark, "Natural Products as Resource of New Drugs," Pharmaceutical Research, Vol. 13, No. 8, 1996, pp. 1133-1141.

[16] G. A. Cordell, "Biodiversity and Drug Discovery a Symbiotic Relationship," Phytochemistry, Vol. 55, No. 66, 2000, pp. 463-480.

[17] J. R. Zgoda and J. R. Porter, "A Convenient Microdilution Method for Screening Natural Products against Bacteria and Fungi," Pharmaceutical Research, Vol. 39, No. 3, 2001, pp. 211-225.

[18] T. J. Walsh, J. W. Hathorn, J. D. Sobel, W. G. Merz, V. Sanchez, S. N. Maret, H. R. Buckley, M. A. Pfaller, R. Schaufele, C. Sliva, E. Navarro, J. Lecciones, P. Chandrasekar, J. Lee and P. A. Pizzo, "Detection of Circulating Candida enolase by Immunoassay in Patients with Cancer and Invasive Candidiasis," New England
Journal of Medicine, Vol. 324, No. 15, 1991, pp. 10261031.

[19] R. M. Herrera, M. Perez, D. A. Martin-Herrera, R. Lopez-Garcia and R. M. Rabanal, "Antimicrobial Activity of Extracts from Plants Endemic to the Canary Islands," Phytotherapy Research, Vol. 10, No. 6, 1996, pp. 364-366.

[20] N. A. A. Ali, W. D. Julich, C. Kusnick and U. Lindequist, "Screening of Yemeni Medicinal Plants for Antibacterial and Cytotoxic Activities," Journal of Ethnopharmacology, Vol. 74, No. 2, 2001, pp. 173-179.

[21] R. Nair and S. Chanda, "In vitro Antimicrobial Activity of Psidium guajava L. Leaf Extracts against Clinically Important Pathogenic Microbial Strains," Brazilian Journal of Microbiology, Vol. 38, No. 3, 2007, pp. 452-458. 